ORIGINAL ARTICLE

\title{
Neonatal resuscitation 2: an evaluation of manual ventilation devices and face masks
}

\author{
C P F O'Donnell, P G Davis, R Lau, P A Dargaville, L W Doyle, C J Morley
}

Arch Dis Child Fetal Neonatal Ed 2005;90:F392-F396. doi: 10.1136/adc.2004.064691

See end of article for authors' affiliations

....................

Correspondence to: Dr O'Donnell, Royal

Women's Hospital

Melbourne, 132 Grattan

Street, Carlton, Victoria

3053, Australia;

colm.odonnell@

rwh.org.au

Accepted 18 March 2005

Published Online First

4 May 2005

Background: The key to successful neonatal resuscitation is effective ventilation. Little evidence exists to guide clinicians in their choice of manual ventilation device or face mask. The expiratory tidal volume measured at the mask ( $\left.\mathrm{V}_{\mathrm{TE}(\mathrm{mask})}\right)$ is a good estimate of the tidal volume delivered during simulated neonatal resuscitation.

Aim: To compare the efficacy of $(a)$ the Laerdal infant resuscitator and the Neopuff infant resuscitator, used with (b) round and anatomically shaped masks in a model of neonatal resuscitation.

Methods: Thirty four participants gave positive pressure ventilation to a mannequin at specified pressures with each of the four device-mask combinations. Flow, inspiratory tidal volume at the face mask ( $\left.\mathrm{V}_{T \mid(m a s k)}\right)$, $V_{T E(m a s k)}$, and airway pressure were recorded. Leakage from the mask was calculated from $V_{T I(m a s k)}$ and $\mathrm{V}_{\mathrm{TE} \text { (mask). }}$

Results: A total of 10780 inflations were recorded and analysed. Peak inspiratory pressure targets were achieved equally with the Laerdal and Neopuff resuscitators. Positive end expiratory pressure was delivered with the Neopuff but not the Laerdal device. Despite similar peak pressures, $\mathrm{V}_{\mathrm{TE}(\text { mask) }}$ varied widely. Mask leakage was large for each combination of device and mask. There were no differences between the masks.

Conclusion: During face mask ventilation of a neonatal resuscitation mannequin, there are large leaks around the face mask. Airway pressure is a poor proxy for volume delivered during positive pressure ventilation through a mask.

I nternational consensus statements ${ }^{12}$ and guidelines from various bodies advise how to resuscitate newborn infants..$^{3-7}$ All agree that the key is effective ventilation and recommend giving positive pressure ventilation (PPV) with manual ventilation devices using face masks. Self inflating bags, flow inflating bags, ${ }^{1-7}$ and $\mathrm{T}$ pieces ${ }^{4}{ }^{7}$ are recommended, but a preference for one type of device is not expressed. It is recommended that the face mask used, whether round or anatomically shaped, should have a cushioned rim. ${ }^{1-7}$

Whereas the manual ventilation devices used to resuscitate newborns vary within countries and world wide, the Laerdal infant resuscitator (Laerdal Medical, Victoria, Australia) appears to be the most commonly used. ${ }^{89}$ Although not described in consensus statements, use of a $\mathrm{T}$ piece-the Neopuff infant resuscitator (Fisher \& Paykel Healthcare, Auckland, New Zealand) - appears common. ${ }^{8}{ }^{9}$ There is more uniformity in the masks used, a clear preference being shown for round masks. ${ }^{8} 9$

There is a dearth of evidence about which device is superior for resuscitating newborns. The only prospective quasirandomised trial compared two self inflating bags. ${ }^{10}$ The few studies of newborns given mask ventilation at delivery reported that tidal volumes sufficient for gas exchange were rarely delivered. ${ }^{11-14}$ An in vitro study reported self inflating bags to be more effective than flow inflating bags in delivering an adequate minute volume. ${ }^{15} \mathrm{~A}$ further in vitro study reported that more consistent airway pressures were delivered with a $\mathrm{T}$ piece than with a flow inflating bag. ${ }^{16}$

In the only examination of face masks to date, participants had little or no experience of neonatal resuscitation, a mechanical ventilator was used to give inflations to well, spontaneously breathing infants, and leakage was not measured. ${ }^{16}$ This study suggested that round masks with a cushioned rim leak less than anatomically shaped masks without a cushioned rim.

We have described a system for measuring leakage from masks and estimating tidal volumes delivered during simulated neonatal resuscitation. ${ }^{17}$ We used this system to evaluate the two ventilation devices and two face masks. The aims of this study were to: (a) compare the Laerdal and Neopuff infant resuscitators in terms of operators' ability to deliver PPV at specified pressures; $(b)$ compare the Laerdal and the Neopuff devices with regard to their ability to deliver tidal volume; (c) compare leakage during PPV with a round face mask and an anatomically shaped mask; $(d)$ determine if greater experience of neonatal resuscitation predicted better tidal volume delivery and less mask leakage; (e) assess whether preference for a manual ventilation device predicted appropriate tidal volumes and low leakage with that device.

\section{MATERIALS AND METHODS \\ Setting}

Staff at the intensive care nursery of the Royal Women's Hospital were invited to participate. All had completed the hospital's neonatal resuscitation training programme before the study. Participants were in four groups: consultants, fellows, residents, and neonatal nurses. The experience in neonatology of each participant was recorded. At completion of the study, each participant was asked to state their preferred device and mask.

\footnotetext{
Abbreviations: PEEP, positive end expiratory pressure; PIP, peak

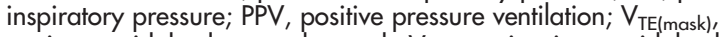

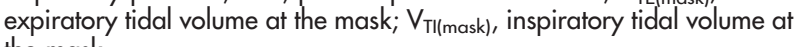
the mask 
Table 1 Years of experience in neonatal paediatrics, and device and mask preferences in each group

\begin{tabular}{lllll}
\hline Group & N & $\begin{array}{l}\text { Years of } \\
\text { experience }\end{array}$ & $\begin{array}{l}\text { Device preference } \\
\text { (Laerdal/Neopuff) }\end{array}$ & $\begin{array}{l}\text { Mask preference } \\
\text { (Round/ } \\
\text { anatomical) }\end{array}$ \\
\hline Consultants & 7 & $17(5-33)$ & $1 / 6$ & $3 / 4$ \\
Fellows & 10 & $3(2-20)$ & $1 / 9$ & $8 / 2$ \\
Residents & 8 & $0.6(0.5-2)$ & $0 / 8$ & $6 / 2$ \\
Nurses & 9 & $7.5(2.8-13)$ & $0 / 9$ & $6 / 3$ \\
\hline \multicolumn{7}{l}{ Values are median (range). } \\
\end{tabular}

\section{Manual ventilation devices}

The Laerdal infant resuscitator is a $240 \mathrm{~m} /$ silicone self inflating bag. Although not supplied as standard with this device, manometers are attached at our hospital, and operators are encouraged to use them. A manometer was used for this study. The Neopuff is a $\mathrm{T}$ piece device that requires a gas source, which was set at 8 litres/min for this study, as is standard in our delivery rooms. This device has a manometer built in and a valve on the outlet which allows a positive end expiratory pressure (PEEP) to be set for a given flow rate. Occlusion of this valve generates a preset peak inspiratory pressure (PIP). Both of these devices are routinely used at our hospital, and all participants were accredited to use both. Flow inflating bags are not routinely used at our hospital and were not used in this study.

\section{Face masks}

Each device was used with both a size 0/1 round Laerdal face mask (Laerdal Medical) and a size 1 anatomically shaped face mask with a partially air filled cushioned rim (Intersurgical, Wokingham, UK). We routinely use the Laerdal mask at our hospital. Participants had not used the anatomically shaped mask before this study.

\section{Model}

We modified a Laerdal Resusci Baby mannequin (Laerdal Medical) by placing a test lung (Dräger, Lubeck, Germany) with a baseline volume of $50 \mathrm{ml}$ in its "thorax". It was connected via an airtight seal to the mannequin's "oropharynx", so that its inflation and deflation caused "chest" excursion similar to that of the unaltered mannequin. A pressure monitoring line was connected immediately proximal to the test lung. This system had a compliance of $0.46 \mathrm{ml} / \mathrm{cm} \mathrm{H}_{2} \mathrm{O}$.

\section{Recording equipment}

We used the Florian Respiratory Monitor (Acutronic Medical Systems, Zug, Switzerland) to measure pressures and gas flow. This monitor measures airway pressures directly and was calibrated against a column of water. It uses a flow sensor to detect gas flow and calculates the volume of gas passing through the sensor by integration of the flow signal. The volume measurement was calibrated with a fixed volume syringe. The output from the Florian monitor was connected through an analogue-digital converter to a computer and acquired using the Spectra software program (Grove Medical, London, UK), a program specifically designed for the acquisition and analysis of respiratory signals.

\section{Values measured}

The airway pressures delivered were measured directly. The flow sensor from the Florian monitor was placed between each ventilation device and mask. The monitor thus calculated the volume of gas passing from the device through the mask-the inspiratory tidal volume at the mask $\left(\mathrm{V}_{\mathrm{TI}(\text { mask })}\right)$-and the volume of gas returning from the mannequin through the mask-the expiratory tidal volume

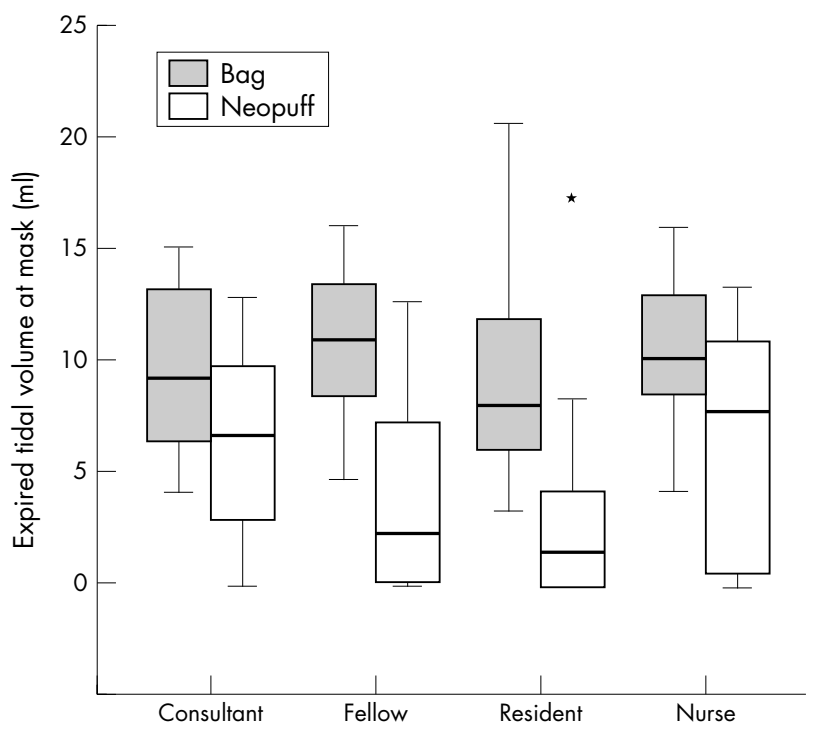

Figure 1 Expiratory tidal volume at the mask by device and group. Box plots show median values (solid bar), interquartile range (margins of box), range of data, and any extreme values (indicated by an asterisk).

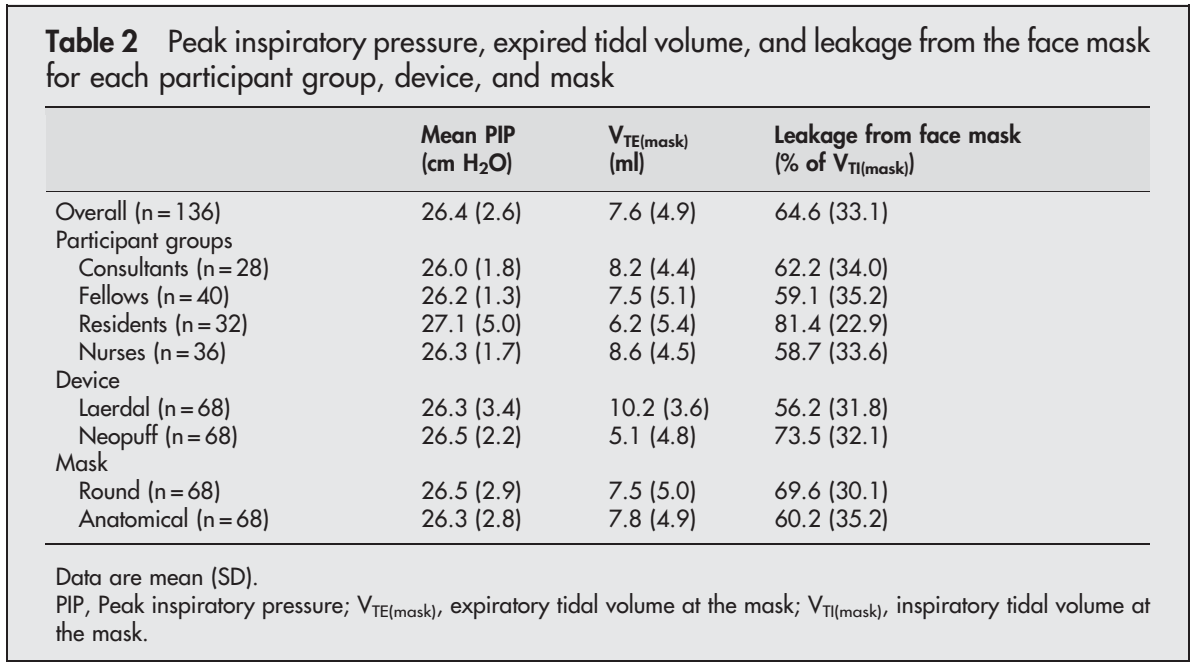


at the mask $\left(\mathrm{V}_{\mathrm{TE} \text { (mask) }}\right)$. We have shown that $\mathrm{V}_{\mathrm{TE} \text { (mask) }}$ is a good estimate of the tidal volume delivered to the test lung in this model. ${ }^{17}$ The flow, volume, and pressure signals for each inflation were examined using LabView (National Instruments, Austin, Texas, USA) software and a program specifically developed by one of the investigators (PAD) for detailed analysis of neonatal respiratory signals.

\section{Values calculated}

The volume of gas leaking at the mask was determined as a percentage of the inspiratory tidal volume at the mask:

$$
\text { leak }(\%)=\left(\left(\mathrm{V}_{\mathrm{TI}(\text { mask })}-\mathrm{V}_{\mathrm{TE}(\text { mask })}\right) / \mathrm{V}_{\mathrm{TI}(\text { mask })}\right) \times 100 \text {. }
$$

\section{Instructions}

Participants gave PPV to the mannequin with combinations of the Laerdal bag, the Neopuff, and both masks. The order in which the four combinations were used was allocated randomly. Participants were requested to ventilate the mannequin for two minutes with PIP $25 \mathrm{~cm} \mathrm{H}_{2} \mathrm{O}$, PEEP $5 \mathrm{~cm} \mathrm{H}_{2} \mathrm{O}$, and to ensure adequate chest excursion. The instructions given were the same for each device-mask combination. Participants could not see the flow, volume, or pressure traces on the Florian monitor or computer.

\section{Statistical analysis}

Data were analysed using SPSS, version 11.5 (SPSS Inc, Chicago, Illinois, USA). Data from participants were averaged for each device and mask combination, yielding data for 136 participant-device-mask combinations. Differences between means were determined by analysis of variance, with the main analyses comparing the two devices, the two masks, and the four participant groups (consultants, fellows, residents, and nurses), allowing all interactions between participant-device-mask combinations. If there were significant differences on overall analysis of variance for participant group, data were analysed post hoc by the least significant difference method to determine between which participant groups the significant differences occurred. Mean differences and $95 \%$ confidence intervals (CIs) were calculated where appropriate. $\mathrm{p}<0.05$ were considered significant.

\section{RESULTS}

\section{Participants}

Thirty four staff members (seven consultants, 10 fellows, eight residents, and nine neonatal nurses) participated in this study (years of experience shown in table 1).

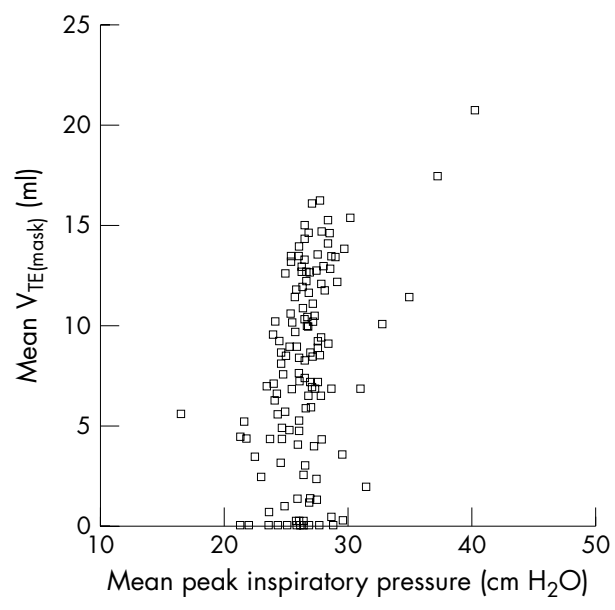

Figure 2 Scatter plot showing relation between mean peak inspiratory pressure and mean expiratory tidal volume at the mask $\left(\mathrm{V}_{\mathrm{TE}(\text { mask })}\right)$.

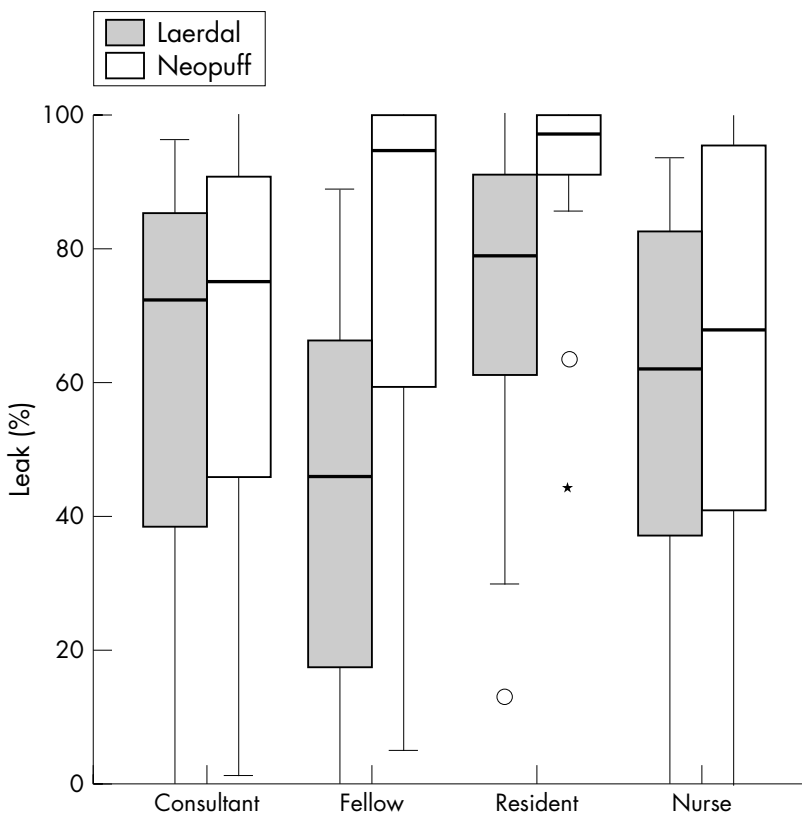

Figure 3 Percentage leakage by device and group. Box plots show median values (solid bar), interquartile range (margins of box), range of data, any outliers (indicated by a circle), and extreme values (indicated by an asterisk).

\section{Measurements}

A total of 10780 inflations were recorded from the 34 participants using each of the four device-mask combinations. The data for each participant group are shown as mean (SD) in table 2.

\section{Peak inspiratory pressure}

Overall, the mean (SD) PIP was $26.4(2.6) \mathrm{cm} \mathrm{H}_{2} \mathrm{O}$. Table 2 shows the mean (SD) for the PIP for each participant group, device, and mask. There was no significant difference for devices $\left(F_{1,130}=0.02, \mathrm{p}=0.88\right)$, participant groups $\left(F_{3,130}=\right.$ $0.82, \mathrm{p}=0.48)$, or masks $\left(F_{1,130}=0.08, \mathrm{p}=0.78\right)($ table 2$)$.

\section{Positive end expiratory pressure (PEEP)}

Participants delivered PEEP with the Neopuff (mean (SD) 4.5 (1.1) $\mathrm{cm} \mathrm{H}_{2} \mathrm{O}$ ), but not the Laerdal bag (mean (SD) 0.0 (1.0) $\mathrm{cm} \mathrm{H}_{2} \mathrm{O}$ ).

\section{Expiratory tidal volume at the mask ( $\left.\mathrm{V}_{\mathrm{TE} \text { (mask) }}\right)$}

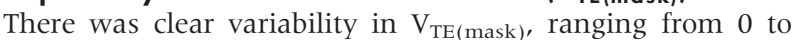

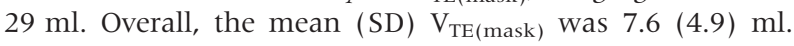
Table 2 shows the mean $\mathrm{V}_{\mathrm{TE} \text { (mask) }}$ for each participant group, device, and mask. The overall $\mathrm{V}_{\mathrm{TE} \text { (mask) }}$ with the Neopuff was lower than with the Laerdal bag $\left(F_{1,130}=50.0, \mathrm{p}<0.001\right.$; table 2, fig 1). There was variation between the different participant groups, but this variation was not significant overall $\left(F_{3,130}=2.1, \mathrm{p}=0.10\right.$; table 2$)$. There was no significant difference between masks $\left(F_{1,130}=0.3, \mathrm{p}=\right.$ 0.60 ; table 2).

\section{Pressure and expired tidal volume}

Although there was a significant positive relation between the mean $\mathrm{V}_{\mathrm{TE} \text { (mask) }}$ delivered and mean PIP used ( $\%$ of variance explained $=13.7, \mathrm{p}<0.001)$, the relation between the two variables was weak. For example, between delivered peak pressures of $20-30 \mathrm{~cm} \mathrm{H}_{2} \mathrm{O}$, the $\mathrm{V}_{\mathrm{TE} \text { (mask) }}$ ranged from as low as zero to as high as $17 \mathrm{ml}$ (fig 2). 


\section{What is already known on this topic}

- PPV of newborns using manual ventilation devices and a mask is one of the most common medical interventions

- Although a variety of devices and masks are available, there is little evidence of efficacy or superiority for any commonly used equipment. These devices are commonly used with manometers to measure the pressures to avoid using excessive pressures and consequent over-distension of the lung

\section{Percentage of gas leak from the face masks}

Overall, the mean (SD) leak between the mask and the mannequin's face was $65(33) \%$. Table 2 shows the mean (SD) leak from the face mask for each participant group, device, and mask. The leak was higher for the Neopuff than the Laerdal $\left(F_{1,130}=10.9, \mathrm{p}=0.001\right.$; table 2 , fig 3$)$. There was variation between the participant groups $\left(F_{3,130}=4.1\right.$, $\mathrm{p}=0.008$; table 2 , fig 3 ). On post hoc analysis, the residents had more leakage than the consultants (mean difference $19.2 \%, 95 \%$ CI $3.5 \%$ to $34.9 \%$ ), the fellows (mean difference $22.3 \%, 95 \%$ CI $7.9 \%$ to $36.6 \%$ ), and the nurses (mean difference $22.7 \%, 95 \%$ CI $7.9 \%$ to $37.4 \%$ ). There was no significant difference between masks $\left(F_{1,130}=3.1, \mathrm{p}=\right.$ 0.08 ; table 2).

\section{Interactions}

There were no significant interactions between participant group, device, or mask for any variable (data not shown).

\section{Participants' preferences}

The Neopuff was preferred by 32 of 34 participants, and most preferred the round mask (table 1). As so few preferred the Laerdal, we decided against comparing performance with the preferred combination of device and mask.

\section{DISCUSSION}

Our study shows that, while giving PPV via a mask, it is possible to deliver highly variable volumes despite generating very similar airway pressures (fig 3 ). This illustrates that airway pressure is a very crude proxy for the volume of gas delivered during ventilation via a mask. Thus clinicians may be falsely reassured that effective ventilation is occurring because the desired pressures are achieved. The aim of ventilation is to provide a volume of gas to the lung adequate for gas exchange. A direct measure of the variable of interest (volume) would be more valuable than the current poor substitute (inflating pressure).

An important difference between the two ventilation devices is the provision of PEEP with the Neopuff. Although there are no current recommendations about the use of PEEP during resuscitation, there are many reasons to believe that it may be beneficial, particularly for very premature infants. ${ }^{18}$ The provision of PEEP with the Neopuff partially explains the smaller $\mathrm{V}_{\mathrm{TE} \text { (mask) }}$ delivered with this device, as the inflating pressure (difference between peak and trough pressure, $20 \mathrm{~cm} \mathrm{H}_{2} \mathrm{O}$ here) is smaller than that with the Laerdal $\left(25 \mathrm{~cm} \mathrm{H}_{2} \mathrm{O}\right)$.

This study demonstrates substantial leakage from the mask during simulated neonatal resuscitation, even in the most experienced hands. In the sole study of face masks, the absence of significant leakage was inferred from the achievement of set mechanical ventilator pressures. ${ }^{16}$ Our finding that a target PIP may be achieved with large leaks

\section{What this study adds}

- During mask ventilation of a neonatal resuscitation mannequin there are large leaks around the mask, irrespective of the device used

- Airway pressure is a poor proxy for volume delivered during PPV via a mask

suggests that this inference is not correct. It has been suggested that resuscitation bags of $240 \mathrm{ml}$ are too small to resuscitate newborns and that larger paediatric (at least $450 \mathrm{ml}$ ) bags should be used. ${ }^{12}$ Given that an infant's tidal volume is about $4-8 \mathrm{ml} / \mathrm{kg}$, a $240 \mathrm{ml}$ bag should be more than adequate. That these devices have been shown to be inadequate ${ }^{13}$ suggests that there was considerable leakage in the system, most likely at the mask. We were surprised that the anatomical mask was no different from the round mask, as this was the first time participants had used it. It is possible that, with experience, performance could improve with this model.

Our findings that a target pressure can be delivered accurately by operators with varying experience using a Neopuff are similar to those of Finer et al. ${ }^{15}$ Somewhat surprisingly, participants also achieved the target PIP accurately with the Laerdal bag. The Laerdal bag is often not used with a manometer, and it may have improved the participants' ability to deliver this target PIP. The manometer of the Neopuff is built into the control box and is some distance from the $\mathrm{T}$ piece. Some participants remarked that they tended to concentrate on the pressures being delivered rather than the mannequin's chest excursion. The manometer in the Laerdal circuit seemed less of a distraction, as most participants placed it alongside the mannequin's chest, allowing simultaneous observation of both the manometer and chest excursion. This may help to explain the smaller leakage and larger $\mathrm{V}_{\mathrm{TE} \text { (mask) }}$ delivered with the Laerdal.

All but two participants expressed a preference for the Neopuff. This precluded meaningful statistical analysis of the effect of operator preference. However, such a clear preference combined with inferior performance suggests that personal preference for a device is not a reliable way to discern the most effective tool for resuscitation.

\section{CONCLUSION}

During face mask ventilation of a neonatal resuscitation mannequin, there are large leaks around the face mask. Widely varying volumes may be delivered with similar target peak airway pressures regardless of the device and mask used or the experience of the resuscitator. This shows that airway pressure is a poor proxy for the tidal volume delivered during positive pressure ventilation via a face mask. Specified peak airway pressures may be generated accurately using a Laerdal bag with a manometer and a Neopuff infant resuscitator, although only the Neopuff produces PEEP. Larger tidal volumes were delivered with the Laerdal bag and less leakage was seen from the face mask than with the Neopuff. Clinical studies to determine the most effective devices and techniques to give PPV to newborns at delivery are urgently needed.

\section{ACKNOWLEDGEMENTS}

CPFO'D is supported in part by a Royal Women's Hospital Postgraduate Degree Scholarship. PAD and PGD were supported in part by the Murdoch Children's Research Institute. PGD is supported by an Australian National Health and Medical Research Council Practitioner Fellowship. 


\section{Authors' affiliations}

C P F O'Donnell, P G Davis, R Lau, L W Doyle, C J Morley, Royal

Women's Hospital Melbourne, Victoria 3053, Australia

P A Dargaville, Royal Hobart Hospital, Hobart, Tasmania 7000, Australia

Competing interests: none declared

\section{REFERENCES}

1 Kattwinkel J, Niermeyer S, Nadkarni V, et al. ILCOR advisory statement: resuscitation of the newly born infant. Pediatrics 1999;103:e56.

2 International guidelines for neonatal resuscitation: an excerpt from the guidelines 2000 for cardiopulmonary resuscitation and emergency cardiovascular care: international consensus on science. Pediatrics 2000;106:e29.

3 Kattwinkel J, ed. Neonatal resuscitation program: textbook of neonatal resuscitation, 4th ed. Washington DC: American Academy of Pediatrics/ American Heart Association, 2000

4 Richmond S, ed. Resuscitation at birth: the newborn life support provider manual. London: Newborn Life Support Working Party, Resuscitation Council (UK), 2001

5 Mackway-Jones K, Molyneux E, Phillips B, Wieteska S, eds. Advanced paediatric life support: the practical approach, 3rd ed. London: BMJ Books, 2001.

6 European Resuscitation Council. Recommendations on resuscitation of babies at birth. Resuscitation 1998;37:103-10.
7 Phillips B, Zideman D, Wyllie J, et al. European Resuscitation Council guidelines 2000 for newly born life support. A statement from the Paediatric Life Support Working Group and approved by the Executive Committee of the European Resuscitation Council. Resuscitation 2001:48:235-9.

8 O'Donnell CPF, Davis PG, Morley CJ. Neonatal resuscitation: review of ventilation equipment and survey of practice in Australia and New Zealand. J Paediatr Child Health 2004;40:208-12.

9 O'Donnell CPF, Davis PG, Morley CJ. Positive pressure ventilation at neonatal resuscitation: review of equipment and international survey of practice. Acta Paediatr 2004;93:583-8.

10 Davies VA, Rothberg AD, Argent AC, et al. A comparison of two resuscitators in the management of birth asphyxia. S Afr J Med 1985;68:19-22.

11 Milner AD, Vyas H, Hopkin IE. Efficacy of facemask resuscitation at birth. BMJ 1984:289:1563-5.

12 Field D, Milner AD, Hopkin IE. Efficiency of manual resuscitators at birth. Arch Dis Child 1986;61:300-2.

13 Hoskyns EW, Milner AD, Hopkin IE. A simple method of face mask resuscitation at birth. Arch Dis Child 1987:62:376-8.

14 Kanter RK. Evaluation of mask-bag ventilation in resuscitation of infants. Am J Dis Child 1987;141:761-3.

15 Finer NN, Rich W, Craft A, et al. Comparison of methods of bag and mask ventilation for neonatal resuscitation. Resuscitation 2001;49:299-305.

16 Palme C, Nystrom B, Tunell R. An evaluation of the efficiency of face masks in the resuscitation of newborn infants. Lancet 1985;1:207-10.

17 O'Donnell CPF, Kamlin COF, Davis PG, et al. Neonatal resuscitation 1: evaluating face mask leakage and estimating tidal volume in a model. Arch Dis Child Fetal Neonatal Ed 2005:90.

18 O'Donnell CPF, Davis PG, Morley CJ. Resuscitation of premature infants: what are we doing wrong and can we do better? Biol Neonate 2003;84:76-82. 